PSS PROCEEDINGS

\title{
Review on low and high mass spectroscopy
}

\author{
Chang-Zheng Yuan* \\ Institute of High Energy Physics, Chinese Academy of Sciences, Beijing 100049, China \\ E-mail: yuancz@ihep.ac.cn
}

We review the most recent experimental progress in the hadron spectroscopy, up to bottomonium mass. This covers the search for the $J^{P C}=1^{-+}$exotic state, the states in $J / \psi$ decays, the spinsinglets of heavy quarkonium, and the charmoniumlike $X Y Z$ states.

35th International Conference of High Energy Physics - ICHEP2010,

July 22-28, 2010

Paris France

* Speaker. 


\section{Introduction}

In the quark model, mesons are the bound state of one quark and one anti-quark, while baryons are composed of three quarks. Although no solid calculation shows hadronic states with other configurations must exist in QCD, people believe hadrons with no quark (glueball), with excited gluon (hybrid), or with more than three quarks (multi-quark state) exist. Since a proton and a neutron can be bounded to form a deuteron, it is also believed that other mesons can also be bounded to form molecules.

It is a long history of searching for all these kinds of states, however, no solid conclusion was reached until now on the existence of any one of them, except deuteron. In this talk, we show the most recent progress in the experimental study of the light and heavy hadrons.

\section{The state with exotic quantum number}

States with exotic quantum numbers that quark and antiquark pair can not have give clear evidence of the existence of non- $q \bar{q}$ states. So far, three candidates with $J^{P C}=1^{-+}$were reported but some of them suffer from controversial experimental information. A partial wave analysis of 2004 COMPASS data [1] from the diffractive dissociation of $190 \mathrm{GeV} \pi^{-}$on a lead target into the $\pi^{-} \pi^{-} \pi^{+}$final state was performed. In addition to well-known $q \bar{q}$ states, a significant spin-exotic wave with $J^{P C}=1^{-+}$decaying to $\rho \pi$ is found. The mass and width are $1660 \pm 10_{-64}^{+0} \mathrm{MeV}$ and $269 \pm 21_{-64}^{+42} \mathrm{MeV}$, respectively. Its mass-dependent phase differences to the $J^{P C}=2^{-+}$and $1^{++}$ waves are consistent with the highly debated $\pi_{1}(1600)$ meson. There are 200 times more events in COMPASS 2008 data, more results on light hadron spectroscopy are expected to come soon.

\section{States in $J / \psi$ decays}

With newly accumulated $106 \mathrm{M} \psi(2 S)$ and $226 \mathrm{M} J / \psi$ events, BESIII experiment [2] confirmed the existence of the threshold enhancement in $J / \psi \rightarrow \gamma p \bar{p}$ final state, with mass agrees with the BESII measurement, and the width less than $8 \mathrm{MeV}$ at the $90 \%$ confidence level (C.L.). In an analysis of the $J / \psi \rightarrow \gamma \eta^{\prime} \pi^{+} \pi^{-}$, three resonances are observed. The $X(1835)$ has a mass of $1838 \pm 3 \mathrm{MeV}$, in good agreement with BESII result, and a width of $180 \pm 9 \mathrm{MeV}$, which is larger than the BESII measurement. The two new structures are at $2124 \mathrm{MeV}$ and $2371 \mathrm{MeV}$, with widthes around $100 \mathrm{MeV}$. In the $\eta \pi^{+} \pi^{-}$invariant mass recoiling against an $\omega$ in $J / \psi$ decays, besides the known $f_{1}(1285)$ and $\eta(1405)$, a state at $1873 \pm 11 \mathrm{MeV}$ with a width of $82 \pm 19 \mathrm{MeV}$ is seen, it could be the hadronic production of the $X(1835)$ observed in $\eta^{\prime} \pi^{+} \pi^{-}$mode, although the mass difference is large. The nature of these states is unknown, with the possibilities of being the excited $\eta$ or $\eta^{\prime}$ states, the glueballs, the $p \bar{p}$ molecular states, and so on.

\section{Spin-singlet charmonium and bottomonium states}

The $P$-wave charmonium spin-singlet state $h_{c}$ is studied at BESIII with $106 \mathrm{M} \psi(2 S)$ events accumulated in 2009 [3, 4]. Clear signals are observed for $\psi(2 S) \rightarrow \pi^{0} h_{c}$ with and without the subsequent radiative decay $h_{c} \rightarrow \gamma \eta_{c}$. First measurements of the absolute branching ratios 
$\mathscr{B}\left(\psi(2 S) \rightarrow \pi^{0} h_{c}\right)=(8.4 \pm 1.3 \pm 1.0) \times 10^{-4}$ and $\mathscr{B}\left(h_{c} \rightarrow \gamma \eta_{c}\right)=(54.3 \pm 6.7 \pm 5.2) \%$ are determined. A statistics limited determination of the previously unmeasured $h_{c}$ width leads to an upper limit $\Gamma\left(h_{c}\right)<1.44 \mathrm{MeV}$ at the $90 \%$ C.L. Measurements of $M\left(h_{c}\right)=3525.40 \pm 0.13 \pm 0.18 \mathrm{MeV}$ and $\mathscr{B}\left(\psi^{\prime} \rightarrow \pi^{0} h_{c}\right) \times \mathscr{B}\left(h_{c} \rightarrow \gamma \eta_{c}\right)=(4.58 \pm 0.40 \pm 0.50) \times 10^{-4}$ are consistent with previous results by CLEOc [7].

Both BaBar [5] and Belle [6] experiments found new decay modes of the radial excitation of the $S$-wave charmonium spin-singlet, the $\eta_{c}(2 S)$, and measured the mass and width of it. The new decay modes are $K^{+} K^{-} \pi^{+} \pi^{-} \pi^{0}, K_{S} K^{+} \pi^{+} \pi^{-} \pi^{-}+$c.c., $K^{+} K^{-} 2\left(\pi^{+} \pi^{-}\right)$, and $3\left(\pi^{+} \pi^{-}\right)$, these add to the previous known modes $K \bar{K} \pi$ and $\gamma \gamma$. The masses are measured to be $3638.3 \pm 1.5 \pm 0.5 \mathrm{MeV}$ (BaBar) and 3636.9 $\pm 1.1 \pm 2.5 \pm 5.0 \mathrm{MeV}$ (Belle), and the widths are 14.2 $\pm 4.4 \pm 2.5 \mathrm{MeV}$ (BaBar) and 9.9 $\pm 3.2 \pm 2.6 \pm 2.0 \mathrm{MeV}$ (Belle). Together with the previous measurements [7], we get the best estimation of the mass and width of the $\eta_{c}(2 S)$ to be $M=3638.0 \pm 1.4 \mathrm{MeV}$ and $\Gamma=12.2 \pm 3.1 \mathrm{MeV}$.

The bottomonium $S$-wave spin-singlet $\eta_{b}$ was observed in $\Upsilon(2 S)$ and $\Upsilon(3 S)$ radiative transitions by BaBar [8] and CLEO [9] experiments. The transition rates are at a few $10^{-4}$ level and the mass splitting from the spin-triplet is $69.3 \pm 2.8 \mathrm{MeV}$. Most recent theoretical calculation gives consistent result but with large uncertainty: $60.3 \pm 5.5$ (stat.) \pm 3.8 (sys.) \pm 2.1 (exp.) $\mathrm{MeV}$ [10]. The calculation predicts the mass splitting of the $2 S$ states of $23.5 \pm 4.1 \pm 1.5 \pm 0.8 \mathrm{MeV}$, however, the observation of $\eta_{b}(2 S)$ should be extremely hard due to the low energy of the radiative photon from $\Upsilon(2 S)$ or $\Upsilon(3 S)$ decays.

BaBar searched for the $P$-wave bottomonium spin-singlet $h_{b}$ in $\Upsilon(3 S) \rightarrow \pi^{+} \pi^{-} h_{b}$ and $\pi^{0} h_{b}$ [11]. No evidence was found in $\pi^{+} \pi^{-} h_{b}$ mode, and $\mathscr{B}\left(\Upsilon(3 S) \rightarrow \pi^{+} \pi^{-} h_{b}\right)<1 \times 10^{-4}$ was determined at the $90 \%$ C.L. There is a faint evidence $(2.7 \sigma)$ at $9903 \mathrm{MeV}$ in $\Upsilon(3 S) \rightarrow \pi^{0} h_{b}$, and the production rate is determined to be $\mathscr{B}\left(\Upsilon(3 S) \rightarrow \pi^{0} h_{b}\right)=(3.1 \pm 1.1 \pm 0.4) \times 10^{-4}$.

\section{The charmoniumlike $X Y Z$ states}

As the $B$-factories accumulate more and more data, lots of new states have been observed in the final states with a charmonium and some light hadrons. All these states populate in the charmonium mass region. They could be candidates for usual charmonium states, however, there are also lots of strange properties shown from these states, these make them more like exotic states rather than conventional charmonium states [12, 13].

\subsection{The $X(3872)$}

The $X(3872)$ was discovered by Belle in $2003[14]$ as a narrow peak in the $\pi^{+} \pi^{-} J / \psi$ invariant mass distribution from $B \rightarrow K \pi^{+} \pi^{-} J / \psi$ decays. With the most precise measurement of the mass of $M_{X(3872)}^{C D F}=3871.61 \pm 0.16 \pm 0.19 \mathrm{MeV}$ [15] from CDF, we know the mass to be $3871.56 \pm 0.22 \mathrm{MeV}$ [7], which is very close to the $D^{* 0} \overline{D^{0}}$ mass threshold: $m_{D^{* 0}}+m_{D^{0}}=$ $3871.78 \pm 0.29 \mathrm{MeV}$ [7]. This suggests a binding energy of $-0.22 \pm 0.36 \mathrm{MeV}$ if $X(3872)$ is interpreted as a $D^{* 0} \overline{D^{0}}$ molecule, to be compared with the binding energy of $-2.2 \mathrm{MeV}$ in deuteron case.

The quantum number of the $X(3872)$ was found to be either $1^{++}$or $2^{-+}$. A study of the $\pi^{+} \pi^{-}$ mass distribution [16] and the observation of its $\gamma J / \psi$ decays [17, 18] indicate that the $C$-parity 
of the $X(3872)$ is even, and the angular correlations among the $\pi^{+} \pi^{-} J / \psi$ final state particles constrains the $J^{P C}$ for the $X(3872)$ to be $1^{++}$or $2^{-+}$, with $1^{++}$preferred [16]. Recently, the $2^{-+}$ assignment was revived by BaBar's analysis of the $\pi^{+} \pi^{-} \pi^{0}$ mass distribution in $\pi^{+} \pi^{-} \pi^{0} J / \psi$ final state, where $2^{-+}$is found to be slightly favored than $1^{++}$[19, 20].

BaBar reported $>3 \sigma$ significance signals for $X(3872)$ decays to both $\gamma J / \psi$ and $\gamma \psi(2 S)$, and the branching fraction of $X(3872) \rightarrow \gamma \psi(2 S)$ is found to be larger than that of $X(3872) \rightarrow \gamma J / \psi[18]$. While at Belle [21], $X(3872) \rightarrow \gamma J / \psi$ was observed with similar production rate as measured by BaBar, but no $X(3872) \rightarrow \gamma \psi(2 S)$ signal was observed, and $\mathscr{B}\left(B^{+} \rightarrow X(3872) K^{+}\right) \times \mathscr{B}(X(3872) \rightarrow$ $\gamma \psi(2 S))<3.4 \times 10^{-6}$ at the $90 \%$ C.L. was determined, smaller than the BaBar's result of $(9.5 \pm$ $2.7 \pm 0.6) \times 10^{-6}[18]$.

BaBar sets an upper limit of the $X(3872)$ production rate in the $B$-meson decays by measuring the momentum distribution of the inclusive kaon from $B$-meson decays [22]: $\mathscr{B}\left(B^{-} \rightarrow\right.$ $\left.K^{-} X(3872)\right)<3.2 \times 10^{-4}$ at the $90 \%$ C.L. Together with all the other measurements on the product branching fractions $\mathscr{B}\left(B^{-} \rightarrow K^{-} X(3872)\right) \cdot \mathscr{B}(X(3872) \rightarrow$ exclusive $)$, one gets $2.3 \%<$ $\mathscr{B}\left(X(3872) \rightarrow \pi^{+} \pi^{-} J / \psi\right)<6.6 \%, 1.4 \times 10^{-4}<\mathscr{B}\left(B^{-} \rightarrow K^{-} X(3872)\right)<3.2 \times 10^{-4}$, at the $90 \%$ C.L. We find that the decay width of the $X(3872)$ to $\pi^{+} \pi^{-} J / \psi$ is larger and the production rate of the $X(3872)$ in $B$ decays is smaller than conventional charmonium states such as $\eta_{c}, \psi(2 S)$, and $\chi_{c 1}$ [7].

\subsection{The $X Y Z$ states near $3.94 \mathrm{GeV}$}

In 2005, Belle reported observations of three states with masses near $3940 \mathrm{MeV}$ : the $X(3940)$, seen as a $D^{*} \bar{D}$ mass peak in $e^{+} e^{-} \rightarrow J / \psi D^{*} \bar{D}$ annihilations [23]; the $Y(3940)$, seen as an $\omega J / \psi$ mass peak in $B \rightarrow K \omega J / \psi$ [24]; and the $Z(3930)$, seen as a $D \bar{D}$ mass peak in $\gamma \gamma \rightarrow D \bar{D}$ [25]. The $Y(3940)$ and $Z(3940)$ were confirmed by BaBar experiment [26, 27].

The $Y(3940)$ mass is well above $D \bar{D}$ or $D^{*} \bar{D}$ mass threshold, but was discovered via its decay to the hidden charm $\omega J / \psi$ final state. This implies an $\omega J / \psi$ partial width that is much larger than expectations for usual charmonium. Recently, BaBar [19, 20] reported a study of $B \rightarrow K \omega J / \psi$ in which the $\omega J / \psi$ invariant mass distribution shows clear evidence for the $X(3872)$ and $Y(3940)$. However, the BaBar values for mass and width of the $Y(3940)$ are both lower than the corresponding values reported by Belle and are more precise: $M=3919.1_{-3.5}^{+3.8} \pm 2.0 \mathrm{MeV}$ (BaBar) compared to $3943 \pm 11 \pm 13 \mathrm{MeV}$ (Belle), and $\Gamma=31_{-8}^{+10} \pm 5 \mathrm{MeV}$ (BaBar) compared to $87 \pm 22 \pm 26 \mathrm{MeV}$ (Belle). Part of the difference might be attributable to the larger data sample used by BaBar (426 $\mathrm{fb}^{-1}$ compared to Belle's $253 \mathrm{fb}^{-1}$ ), which enabled them to use smaller $\omega J / \psi$ mass bins as well as considering $X(3872)$ and $Y(3940)$ simultaneously in their analysis.

To add more information to the states in this mass region, Belle observed a dramatic and rather narrow peak, $X(3915)$, in $\gamma \gamma \rightarrow \omega J / \psi[28]$ that is consistent with the mass and width reported for the $Y(3940)$ by the BaBar group. The resonance parameters of the $X(3915)$ are $M=3914 \pm 4 \pm$ $2 \mathrm{MeV}$ and $\Gamma=28 \pm 12_{-8}^{+2} \mathrm{MeV}$, with a statistical significance of $7.1 \sigma$.

Of these, only the $Z(3930)$ has been assigned to a $2^{3} P_{2} c \bar{c}$ charmonium state, which is commonly called the $\chi_{c 2}(2 P)$. If not exotic states, the $X(3940)$ could be a candidate for $\eta_{c}(3 S)$ and the $Y(3940)$ (maybe the same as the $X(3915)$ ) could be $\chi_{c 0}(2 P)$. 


\subsection{The $Y(4140), Y(4280)$, and $X(4350)$}

Using exclusive $B^{+} \rightarrow J / \psi \phi K^{+}$decays, CDF Collaboration observed a narrow structure, $Y(4140)$, near the $J / \psi \phi$ mass threshold with a statistical significance of 3.8 $\sigma$ [29]. The analysis is updated with more data and the signal significance is larger than $5 \sigma$ now [30]. The mass and width of this structure are fitted to be $4143.4_{-3.0}^{+2.9}$ (stat) \pm 0.6 (syst) $\mathrm{MeV}$ and $15.3_{-6.1}^{+10.4}$ (stat) \pm 2.5 (syst) $\mathrm{MeV}$ respectively. Furthermore, there is another narrow structure, $Y(4280)$, with a significance of $3.1 \sigma$ observed with $M=4274.4_{-6.1}^{+10.4}$ (stat) \pm 1.9 (syst) $\mathrm{MeV}$ and $\Gamma=32.3_{-15.3}^{+21.9}$ (stat) \pm 7.6(syst) MeV. They are isospin singlet states with positive $C$ and $G$ parities since the quantum numbers of both $J / \psi$ and $\phi$ are $I^{G}\left(J^{P C}\right)=0^{-}\left(1^{--}\right)$. It was argued by the CDF Collaboration that the $Y(4140)$ can not be a conventional charmonium state, because a charmonium state with mass about $4143 \mathrm{MeV}$ would dominantly decay into open charm pairs, and the branching fraction into the double OZI forbidden modes $J / \psi \phi$ or $J / \psi \omega$ would be negligible.

The Belle Collaboration searched for this state using the same process with $772 \times 10^{6} B \bar{B}$ pairs. No significant signal was found, and the upper limit on the production rate $\mathscr{B}\left(B^{+} \rightarrow Y(4140) K^{+}\right.$, $Y(4140) \rightarrow J / \psi \phi)$ is measured to be $6 \times 10^{-6}$ at the $90 \%$ C.L. Although this upper limit is lower than the central value of the CDF measurement $(7.7 \pm 3.7) \times 10^{-6}$, they do not contradict with each other considering the large error [31].

Assuming the $Y(4140)$ is a $D_{s}^{*+} D_{s}^{*-}$ molecule with quantum number $J^{P C}=0^{++}$or $2^{++}$, the authors of Ref. [32] predicted a two-photon partial width of the $Y(4140)$ of the order of $1 \mathrm{keV}$, which is large and can be tested with experimental data. The Belle Collaboration searched for this state in two-photon process [33]. No $Y(4140)$ signal is observed, and the upper limit on the product of the two-photon decay width and branching fraction of $Y(4140) \rightarrow \phi J / \psi$ is measured to be $\Gamma_{\gamma \gamma}(Y(4140)) \mathscr{B}(Y(4140) \rightarrow \phi J / \psi)<39 \mathrm{eV}$ for $J^{P}=0^{+}$, or $<5.7 \mathrm{eV}$ for $J^{P}=2^{+}$at the $90 \%$ C.L. The upper limit is lower than the predictions in Ref. [32]. This disfavors the scenario of the $Y(4140)$ being a $D_{s}^{*+} D_{s}^{*-}$ molecule with $J^{P C}=0^{++}$or $2^{++}$.

Evidence is reported for a narrow structure at $4.35 \mathrm{GeV} / c^{2}$ in the $\phi J / \psi$ mass spectrum in the above two-photon process $\gamma \gamma \rightarrow \phi J / \psi$ in Belle experiment [33]. A signal of $8.8_{-3.2}^{+4.2}$ events, with statistical significance of greater than 3.2 standard deviations, is observed. The mass and natural width of the structure (named as $X(4350)$ ) are measured to be $4350.6_{-5.1}^{+4.6}$ (stat) \pm 0.7 (syst) $\mathrm{MeV}$ and $13.3_{-9.1}^{+17.9}$ (stat) \pm 4.1 (syst) $\mathrm{MeV}$, respectively. It is noted that the mass of this structure is well consistent with the predicted values of a $c \bar{c} s \bar{s}$ tetraquark state with $J^{P C}=2^{++}$in Ref. [34] and a $D_{s}^{*+} D_{s 0}^{*-}$ molecular state in Ref. [35].

\subsection{The $Y$ states in $I S R$ processes}

The study of charmonium states via initial state radiation (ISR) at the $B$-factories has proven to be very fruitful. In the process $e^{+} e^{-} \rightarrow \gamma_{I S R} \pi^{+} \pi^{-} J / \psi$, the BaBar Collaboration observed the $Y(4260)$ [36]. This structure was also observed by the CLEO [37] and Belle Collaborations [38] with the same technique; moreover, there is a broad structure near $4.008 \mathrm{GeV}$ in the Belle data. In a subsequent search for the $Y(4260)$ in the $e^{+} e^{-} \rightarrow \gamma_{I S R} \pi^{+} \pi^{-} \psi(2 S)$ process, BaBar found a structure at around $4.32 \mathrm{GeV}$ [39], while the Belle Collaboration observed two resonant structures at $4.36 \mathrm{GeV}$ and $4.66 \mathrm{GeV}$ [40]. Recently, CLEO collected $13.2 \mathrm{pb}^{-1}$ of data at $\sqrt{s}=4.26 \mathrm{GeV}$ and 
investigated 16 decay modes with charmonium or light hadrons [41]. The large $e^{+} e^{-} \rightarrow \pi^{+} \pi^{-} J / \psi$ cross section at this energy is confirmed.

Belle and $\mathrm{BaBar}$ have exploited ISR to make measurements of cross sections for exclusive open-charm final states in this energy range [42, 43, 44, 45, 46, 47]. The exclusive channels that have been measured so far nearly saturates the total inclusive cross section, but there is no evidence for peaking near the masses of the $Y$ states. The one exception is $e^{+} e^{-} \rightarrow \Lambda_{c}^{+} \Lambda_{c}^{-}$, which has a threshold peak in the vicinity of the $Y(4660)$ peak mass [44].

The cross sections of charm strange meson pair production $\left(\sigma\left(e^{+} e^{-} \rightarrow D_{s}^{+} D_{s}^{-}\right), \sigma\left(e^{+} e^{-} \rightarrow\right.\right.$ $D_{s}^{+*} D_{s}^{-}+$c.c. $)$and $\left.\sigma\left(e^{+} e^{-} \rightarrow D_{s}^{+*} D_{s}^{-*}\right)\right)$ were measured by BaBar [48] and Belle [49] recently. However, the data show no indication of any of the $Y$ states, as in the non-strange charmed meson pair final states.

The absence of any evidence for the $Y(4260)(Y(4360))$ decays to open charm implies that the $\pi^{+} \pi^{-} J / \psi\left(\pi^{+} \pi^{-} \psi(2 S)\right)$ partial width is large: the analysis of Ref. [50] gives a 90\% C.L. lower limit $\Gamma\left(Y(4260) \rightarrow \pi^{+} \pi^{-} J / \psi\right)>508 \mathrm{keV}$, which should be compared with the corresponding $\pi^{+} \pi^{-} J / \psi$ partial widths of established $1^{--}$charmonium states: $201 \mathrm{keV}$ for the $\psi(2 S)$ and $52.7 \mathrm{keV}$ for the $\psi(3770)$ [7].

The nature of these states is unknown, and many possibilities have been proposed [12], including charmonium, molecule, hybrid, baryonium, and so on. There are also argument that these structures are purely threshold effect or the final state interaction of the charmed meson pairs. More experimental measurements and more theoretical calculations are badly needed to have a deeper understanding of these structures.

\subsection{Multiple solutions in fitting $R$-values [51]}

In explaining the $Y$ states and the excited $\psi$ states, the leptonic partial widths provide very important information. As we know, the vector quarkonium states could be either $S$-wave or $D$-wave spin-triplet states, with the $S$-wave states couple strongly to lepton pair while the $D$-wave states couple weakly since the latter are only proportional to the second derivative of the wave-function at the origin squared, as expected in the potential models. This leads people to believe that the $\psi(4040)$ is the $3 S$ charmonium state, $\psi(4160)$ the $2 D$ state, and $\psi(4415)$ the $4 S$ state. This has been a well accepted picture for more than two decades before the discovery of the so-called $Y$ particles, namely, the $Y(4008), Y(4260), Y(4360)$, and $Y(4660)$. With seven states observed between $4.0 \mathrm{GeV} / c^{2}$ and $4.7 \mathrm{GeV} / c^{2}$, some people started to categorize some of these as non-conventional quarkonium states, while others tried to accommodate all of them in modified potential models. Many of the theoretical models use the leptonic partial widths of these states to distinguish them between $S$ - and $D$-wave assumptions [52, 53], and most of the time, the values on the leptonic partial widths are cited from the PDG [7] directly. Although the resonance parameters of these excited $\psi$ states have been measured by many experimental groups, all of them were obtained by fitting the $R$-values measured in the relevant energy region. The most recent ones [54], which were from a sophisticated fit to the most precise $R$-values measured by the BES collaboration [55, 56], are the only source of the leptonic partial widths of these three $\psi$ states now quoted by the PDG [7].

In fitting to the BES data, unlike the previous analyses, the BES collaboration considered the interference between the three resonances decaying into the same final modes, and introduced a free relative phase for the amplitude of each resonance [54]. The new parametrization of the hadronic 
Table 1: Four groups of solutions for the data fitting. The four solutions have identical resonance masses $(M)$ and total widths $\left(\Gamma_{t}\right)$, but significant different leptonic partial widths $\left(\Gamma_{e e}\right)$ and the relative phases $(\phi)$.

\begin{tabular}{cccc}
\hline \hline Parameter & $\psi(4040)$ & $\psi(4160)$ & $\psi(4415)$ \\
\hline$M(\mathrm{MeV})$ & $4034 \pm 6$ & $4193 \pm 7$ & $4412 \pm 15$ \\
$\Gamma_{t}(\mathrm{MeV})$ & $87 \pm 11$ & $79 \pm 14$ & $118 \pm 32$ \\
\hline \hline$\Gamma_{e e}^{(1)}(\mathrm{keV})$ & $0.66 \pm 0.22$ & $0.42 \pm 0.16$ & $0.45 \pm 0.13$ \\
$\phi^{(1)}($ radian$)$ & 0 (fixed) & $2.7 \pm 0.8$ & $2.0 \pm 0.9$ \\
\hline$\Gamma_{e e}^{(2)}(\mathrm{keV})$ & $0.72 \pm 0.24$ & $0.73 \pm 0.18$ & $0.60 \pm 0.25$ \\
$\phi^{(2)}($ radian$)$ & 0 (fixed) & $3.1 \pm 0.7$ & $1.4 \pm 1.2$ \\
\hline$\Gamma_{e e}^{(3)}(\mathrm{keV})$ & $1.28 \pm 0.45$ & $0.62 \pm 0.30$ & $0.59 \pm 0.20$ \\
$\phi^{(3)}($ radian$)$ & 0 (fixed) & $3.7 \pm 0.4$ & $3.8 \pm 0.8$ \\
\hline$\Gamma_{e e}^{(4)}(\mathrm{keV})$ & $1.41 \pm 0.12$ & $1.10 \pm 0.15$ & $0.78 \pm 0.17$ \\
$\phi^{(4)}($ radian$)$ & 0 (fixed) & $4.1 \pm 0.1$ & $3.2 \pm 0.3$ \\
\hline \hline
\end{tabular}

cross section results in a pronounced increase of the $\psi(4160)$ mass, and significant decrease of the leptonic partial widths of $\psi(4160)$ and $\psi(4415)$.

In a recent study [51], it is pointed out multiple solutions can be found in the fit to the $R$-values, resulting four sets of solutions for the leptonic partial widths of the excited $\psi$ states. The fit results are presented in Table 1, there are four solutions found in the fit. It should be noted that the four solutions have identical $\chi^{2}$, masses, and total widths for the resonances, but different partial widths to lepton pairs.

As the $\Gamma_{e e}$ of the vector resonances are closely related to the nature of these states, the choice among the distinctive solutions affects the classification of the charmonium and charmonium-like states observed in this energy region. The $Y(4260)$ was proposed to be the $\psi(4 S)$ state and the $\psi(4415)$ be $\psi(5 S)$ in Refs. [52, 53], we can see that in this assignment, the calculated partial widths of $\psi(4040)=\psi(3 S)$ and $\psi(4415)=\psi(5 S)$ [53] agree well with the fourth solution listed in Table 1 .

Of course the possible mixing between $S$ - and $D$-wave states will change significantly the theoretical predictions of the partial widths of these states [58], and the QCD correction, which is not well handled [57], may also change the theoretical predictions significantly. So far, we have no concrete criteria to choose any one of the solutions as the physics one.

It should be noticed that if the $Y$ states are considered together with the excited $\psi$ states in fitting the $R$-values, there could be even more solutions, the situation may become more complicated. We also notice that the existence of the multiple solutions is due to the inclusion of a free phase between two resonances, if these phases can be determined by other means (either theoretically or experimentally), it will be very helpful to know which solution corresponds to the real physics.

\section{Summary}

In summary, there are lots of progress in low and high mass spectroscopy. A better under- 
standing of the charmonium and bottomonium spin-singlets is achieved, although $h_{b}$ is still not observed. There are lots of new states, composed of either only light quarks or light and heavy quarks, observed in experiments. Some of these states have very exotic properties, which may suggest the long searching exotic states have been observed. However, due to limited statistics, the experimental information on the properties of any of these states is not enough for us to draw solid conclusion, let alone our poor knowledge on the QCD prediction of the properties of the exotic states or the conventional mesons.

In the near future, BESIII experiment [59] may accumulate more data for center of mass energy between 3.0 and $4.6 \mathrm{GeV}$, this will contribute to the understanding of some of the states discussed above; the Belle II experiment [60] under construction, with about $50 \mathrm{ab}^{-1}$ data accumulated, will surely improve our understanding of all these states. We also expect new results from hadron colliders as well as fixed target experiments.

\section{Acknowledgment}

We thank the organizers for their kind invitation and congratulate them for a successful conference. This work is supported in part by National Natural Science Foundation of China under Contract Nos. 10825524 and 10935008.

\section{References}

[1] M. G. Alekseev et al. (COMPASS Collaboration), Phys. Rev. Lett. 104 (2010) 241803; F. Nerling, these proceedings.

[2] Y. P. Huang, these proceedings.

[3] M. Ablikim et al. (BESIII Collaboration), Phys. Rev. Lett. 104 (2010) 132002.

[4] G. Li, these proceedings.

[5] V. Druzhinin, these proceedings.

[6] H. Nakazawa, these proceedings.

[7] K. Nakamura et al. (Particle Data Group), J. Phys. G 37 (2010) 075021.

[8] B. Aubert et al. (BaBar Collaboration), Phys. Rev. Lett. 101 (2008) 071801 [Erratum-ibid. 102 (2009) 029901]; Phys. Rev. Lett. 103 (2009) 161801.

[9] G. Bonvicini et al. (CLEO Collaboration), Phys. Rev. D 81 (2010) 031104.

[10] S. Meinel, these proceedings, and arXiv:1007.3966.

[11] B. Fulsom, these proceedings.

[12] See, for example, a recent review by the Quarkonium Working Group, arXiv:1010.5827 [hep-ph].

[13] C. Z. Yuan, arXiv:0910.3138 [hep-ph].

[14] S.-K. Choi et al. (Belle Collaboration), Phys. Rev. Lett. 91 (2003) 262001.

[15] A. Abulencia et al. (CDF Collaboration), Phys. Rev. Lett. 103 (2009) 152001.

[16] A. Abulencia et al. (CDF Collaboration), Phys. Rev. Lett. 98 (2007) 132002. 
[17] I. Adachi et al. (Belle Collaboration), hep-ex/0505037.

[18] B. Aubert et al. (BaBar Collaboration), Phys. Rev. Lett. 102 (2009) 132001.

[19] P. del Amo Sanchez et al. (BaBar Collaboration), Phys. Rev. D 82 (2010) 011101.

[20] A. G. Mokhtar, these proceedings.

[21] I. J. Watson, these proceedings.

[22] B. Aubert et al. (BaBar Collaboration), Phys. Rev. Lett. 96 (2006) 052002.

[23] K. Abe et al. (Belle Collaboration), Phys. Rev. Lett. 98 (2007) 082001.

[24] S.-K. Choi et al. (Belle Collaboration), Phys. Rev. Lett. 94 (2005) 182002.

[25] S. Uehara et al. (Belle Collaboration), Phys. Rev. Lett. 96 (2006) 082003.

[26] B. Aubert et al. (BaBar Collaboration), Phys. Rev. Lett. 101 (2008) 082001.

[27] B. Aubert et al. (BaBar Collaboration), Phys. Rev. D 81 (2010) 092003.

[28] S. Uehara et al. (Belle Collaboration), Phys. Rev. Lett. 104 (2010) 092001.

[29] T. Aaltonen et al. (CDF Collaboration), Phys. Rev. Lett. 102 (2009) 242002.

[30] K. Yi, these proceedings.

[31] My own estimation with numbers in Ref. [30].

[32] T. Branz, T. Gutsche, and V. E. Lyubovitskij, Phys. Rev. D 80 (2009) 054019.

[33] C. P. Shen et al. (Belle Collaboration), Phys. Rev. Lett. 104 (2010) 112004.

[34] F. Stancu, J. Phys. G 37 (2010) 075017 [arXiv:0906.2485 [hep-ph]].

[35] Jian-Rong Zhang and Ming-Qiu Huang, arXiv:0905.4672.

[36] B. Aubert et al. (BaBar Collaboration). Phys. Rev. Lett. 95 (2005) 142001.

[37] Q. He et al. (CLEO Collaboration). Phys. Rev. D 74 (2006) 091104(R).

[38] C. Z. Yuan et al. (Belle Collaboration). Phys. Rev. Lett. 99 (2007) 182004.

[39] B. Aubert et al. (BaBar Collaboration). Phys. Rev. Lett. 98 (2007) 212001.

[40] X. L. Wang et al. (Belle Collaboration). Phys. Rev. Lett. 99 (2007) 142002.

[41] T. E. Coan et al. (CLEO Collaboration). Phys. Rev. Lett. 96 (2006) 162003.

[42] G. Pakhlova et al. (Belle Collaboration), Phys. Rev. Lett. 98 (2007) 092001.

[43] G. Pakhlova et al. (Belle Collaboration), Phys. Rev. D 77 (2007) 011103.

[44] G. Pakhlova et al. (Belle Collaboration), Phys. Rev. Lett. 101 (2008) 172001.

[45] G. Pakhlova et al. (Belle Collaboration), Phys. Rev. Lett. 100 (2008) 062001.

[46] G. Pakhlova et al. (Belle Collaboration), Phys. Rev. D 80 (2009) 091101.

[47] B. Aubert et al. (BaBar Collaboration). Phys. Rev. D 76 (2007) 111105; Phys. Rev. D 79 (2007) 092001.

[48] P. del Amo Sanchez et al. (BaBar Collaboration), Phys. Rev. D 82 (2010) 052004; J. Izen, these proceedings. 
[49] G. Pakhlova et al. (Belle Collaboration), arXiv:1010.4397 [hep-ex].

[50] X. H. Mo et al., Phys. Lett. B 640 (2007) 182.

[51] X. H. Mo, C. Z. Yuan, and P. Wang, Phys. Rev. D 82 (2010) 077501.

[52] E. Klempt and A. Zaitsev, Phys. Rept. 454 (2007) 1.

[53] B. Q. Li and K. T. Chao, Phys. Rev. D 79 (2009) 094004.

[54] M. Ablikim et al. (BES Collaboration), Phys. Lett. B 660 (2008) 315.

[55] J. Z. Bai et al. (BES Collaboration), Phys. Rev. Lett. 84 (2000) 594.

[56] J. Z. Bai et al. (BES Collaboration), Phys. Rev. Lett. 88 (2002) 101802.

[57] H.-F. Fu, X.-J. Chen and G.-L. Wang, arXiv:1006.3898 [hep-ph].

[58] A. M. Badalian, B. L. G. Bakker and I. V. Danilkin, Phys. Atom. Nucl. 72 (2009) 638.

[59] D. M. Asner et al., "Physics at BESIII ", arXiv:0809.1869.

[60] SuperKEKB Task Force, KEK Report 2004-4. 